\title{
Atitude frente à dor em trabalhadores de atividades ocupacionais distintas: uma aproximação da psicologia cognitivo-comportamental
}

\author{
Attitude of workers with chronic pain in \\ different occupational activities: an approach \\ of the cognitive-behaviorist psychology
}

Mônica A. G. de Lima 1

Robson Neves 2

Sylvia Sá 3

Cibele Pimenta 4

\footnotetext{
1 Departamento de Medicina Preventiva da FAMEB/UFBA.

Av. Reitor Miguel Calmon, $\mathrm{s} / \mathrm{n}$, Vale do Canela, 40110-100, Salvador BA. monicangelim@terra.com.br 2 Escola de Fisioterapia da Universidade Católica do Salvador.

3 Centro de Estudos de Saúde dos Trabalhadores, CESAT/BA.

4 Escola de Enfermagem da Universidade de São Paulo, USP.
}

Abstract This study presents the attitude of workers in different activities (bank workers, checkout workers and industrial workers) with chronic pain related to the Repetition Strain Injures (RSI) diagnosed in an occupational outpatient clinic - Centro de Estudos da Saúde do Trabalhador (CESAT), in the State of Bahia, Brazil. Sixty five workers from a convenience sample were interviewed, using the short version of the Survey of Pain Attitude, adapted to the Portuguese language. This questionnaire contains seven domains: control, emotion, disability, physical impairment, medication, solicitude, medical cure. Student's test and Mann-Whitney test were used for comparing two and more than two means, respectively. Physical impairment was more important among male industrial workers with less limitation to work. The emotion and control domains were more favorable among people who practice a religion. These results suggest the utilization of this approach to better understand the complexity of the chronic pain among workers, and the necessity of expanding the concepts of assistance and rehabilitation in the field of Occupational Health, through an integral care model.

Key words Chronic pain, Repetition strain injuries, Popular believes, Occupational health
Resumo Este estudo apresenta o perfil da atitude frente à dor em trabalhadores inseridos em atividades ocupacionais distintas com diagnóstico de LER/DORT acompanhados pelo Centro de Estudos da Saúde do Trabalhador do Estado da Bahia (CESAT/Ba). Foram entrevistados 65 trabalhadores numa amostra de conveniência. Utilizou-se como instrumento o Inventário de Atitudes Frente à Dor, na versão reduzida IAD, adaptado para lingua portuguesa, contando com sete domínios ou escalas: cura médica, controle, solicitude, incapacidade, medicação, emoção e dano físico. $\mathrm{Na}$ análise procedeu-se a comparação das médias das escalas do IAD-breve entre as atividades ocupacionais e as variáveis socioculturais. A comparação das escalas entre as atividades ocupacionais, sexo e limitação para o trabalho revela diferenças entre as médias do domínio dano físico numa direção mais desfavorável para os industriários, sexo masculino e menor limitação para o trabalho e nos domínios emoção e controle mais favorável entre os praticantes de religião. Esses achados apontam para a possibilidade de utilização dessa abordagem para uma melhor compreensão da complexidade do fenômeno da dor crônica, assim como a necessidade de expandir o conceito da assistência e reabilitação desses trabalhadores tendo como referência um modelo de cuidado integral. Palavras-chave Dor crônica, LER/DORT, Crenças, Saúde do trabalhador 


\section{Introdução}

O presente estudo apresenta o perfil de atitude frente à dor em trabalhadores inseridos em atividades ocupacionais distintas com diagnóstico de LER/DORT acompanhado pelo Centro de Estudos da Saúde do Trabalhador do Estado da Bahia (CESAT/BA). A iniciativa é parte de reflexões sobre o cuidado integral a esses trabalhadores que trazem em sua história a dor crônica e a incapacidade como elementos centrais de sofrimento e exclusão social implicando mudanças nas suas relações com o trabalho e com sua vida cotidiana.

A magnitude e gravidade dos casos de LER/DORT diagnosticados e acompanhados nos centros de referência à saúde dos trabalhadores de todo o País têm colocado esse agravo como prioritário no campo da Vigilância à Saúde do Trabalhador, com a proposição e implantação de ações voltadas para o conhecimento dos ambientes de trabalho e para a assistência e reabilitação, constituindo-se um tema que agrega inúmeros interesses e diversas formas de ação. A assistência e o cuidado têm promovido a aproximação dos Centros de Referência da Saúde dos Trabalhadores (CRST) de práticas terapêuticas voltadas para o cuidado da dor e incapacidade, agregando progressivamente à atividade diagnóstica e de vigilância de ambientes de trabalho atividades terapêuticas e de reabilitação.

A dor crônica tem sido objeto de estudo de vários campos disciplinares e, há aproximadamente quatro décadas, motivou a organização de uma especialidade médica, multiprofissional e interdisciplinar, constituída a partir do desenvolvimento da Teoria de Comporta. Esta teoria, elaborada por dois neurofisiologistas em meados da década de 1960, propõe pensar a multidimensionalidade da modulação do sistema nervoso central, agregando as experiências passadas (emoção, cognição, motricidade) e várias sensações (cheiro, audição, tato) como elementos que contribuem para a modulação, percepção e resposta à dor (Melzack \& Wall, 1965).

A dor crônica, entendida como uma experiência sensorial e emocional desagradável relacionada com o dano real ou potencial de algum tecido ou que se descreve em termos de tal dano (Associação Internacional para o Estudo da Dor, IASP, 1985), tem sido estudada pela psicologia comportamental e mais recentemente pela psicologia cognitivo-comportamental (Pimenta, 1999; Jensen, 1987), mostrando resulta- dos coerentes com a concepção de que aspectos psicossocioculturais, entre eles os relacionados à atitude, influenciam no modo de o doente perceber, expressar e lidar com a dor (Pimenta, 1999).

No campo das ciências sociais a dor é bastante reconhecida como uma experiência apropriada socioculturalmente ao revelar diferenças no perfil de expressão de dor em grupos étnicos distintos (Zoborowski, 1969; Zola, 1966), a valorização de sintomas e utilização de recursos terapêuticos em contextos culturais distintos (Kleinman et al. 1978; Kleinman, 1987), e a influência do convívio familiar no processo de aculturação especialmente ocorrido na infância, influenciando a conformação de variados perfis de atitude diante da dor (Bates et al. 1993; 1995; Bates \& Lesley, 1994; Helman, 1994).

Estudos transculturais em dor chamam atenção sobre as diferenças qualitativas e quantitativas da experiência da dor em contextos socioculturais distintos, especialmente em relação à reação à dor e ao comportamento de dor. Sua análise através de estudos epidemiológicos, antropológicos e da psicologia comportamental enfatiza a importância de aprofundar a abordagem de questões transculturais no sentido de entender o que chamam de the "big picture" of the human condition in regard to pain and suffering (Moore \& Brødsgaard, 1999). Essa pretensão de compreender singularidades culturais sobre a percepção-expressão da dor e seus impactos sociais é justificada pela possibilidade de identificar e preservar características étnicas e situações locais específicas assim como evitar erros sistemáticos e resultados inválidos.

A psicologia cognitivo-comportamental fundamenta-se no pressuposto de que os processos cognitivos, biológicos, comportamentais, socioculturais e ambientais influenciam-se mutuamente, visando promover o melhor ajustamento entre o indivíduo e seu ambiente. Autores desta abordagem têm elaborado instrumentos para avaliar crenças, atitudes e comportamentos em sujeitos com dor crônica. Dentre esses, o Inventário de Atitude Frente a Dor (Jensen et al. 1987; 1994; Jensen \& Karoly, 1992 tem tido ampla divulgação e foi recentemente traduzido e validado no Brasil (Pimenta, 1999).

Este estudo pretende comparar as variações das escalas de atitude frente à dor, em trabalhadores com diagnóstico de LER/DORT inseridos em atividades ocupacionais distintas (caixas bancários, operadores de caixa de supermercado e industriários), quanto às variáveis socio- 
culturais, à incapacidade para o trabalho e à situação de litígio trabalhista e ou previdenciário.

A escolha de atividades ocupacionais distintas de diferentes processos produtivos, com diferentes perfis de exposição ocupacional a cargas ergonômicas, escolaridade e renda, sustenta-se na hipótese de que essas diferenças possam influenciar no perfil de crenças identificadas pelo inventário de atitude frente à dor, possibilitando revelar heterogeneidades que permitem a organização de ações de cuidado culturalmente sensíveis e voltadas para diferentes necessidades dessa população.

\section{Material e método}

Foram convidados para compor o grupo de estudo a totalidade de trabalhadores caixas bancários, operadores de caixa e industriários atendidos no ambulatório do CESAT nos anos de 1999 e 2000 através de telefonemas e cartas. O projeto foi submetido e aprovado pelo Comitê de Ética da Secretaria Estadual de Saúde da Bahia (SESAB).

Dos 127 trabalhadores com diagnóstico de LER/DORT convidados, 65 responderam positivamente ao convite e compuseram a amostra de conveniência (22 caixas bancários, 18 operadores de caixa e 25 industriários).

Utilizou-se, como instrumento, um questionário estruturado específico e o Inventário de Atitudes Frente à Dor, na versão reduzida IADbreve, proposta por Tait \& Chibnall (1997) adaptado para língua portuguesa (Pimenta, 1999). O questionário foi organizado em duas partes: a primeira referente aos dados de identificação (nome, sexo, matrícula no serviço), características socioculturais (procedência, escolaridade, religião e renda) e caracterização da exposição ocupacional (ocupação atual e anterior, tempo de trabalho, tempo de exposição à sobrecarga ergonômica); e a segunda parte voltada para as características da dor e incapacidade para o trabalho (etiologia, duração, intensidade, mapa corporal, avaliação do sono e de tender points, limitação para o trabalho, a atividade doméstica, a higiene pessoal, o lazer e a atividade sexual). A intensidade da dor foi avaliada pela escala numérica de 0-10 (sendo $0=$ nenhuma dor e $10=$ a pior dor imaginável).

O Inventário de Atitude Frente à Dor (IAD) (Jensen et al., 1987) foi proposto como instrumento para avaliar as atitudes frente à dor de doentes com dor crônica não-oncológica (Pi- menta, 1999) e contém 30 itens correspondentes a sete domínios ou escalas (cura médica, controle, solicitude, incapacidade, medicação, emoção e dano físico). Cada domínio constitui-se de um conjunto de assertivas que são submetidas ao entrevistado solicitando que avalie seu grau de concordância com cada uma, utilizando-se uma escala do tipo Likert de cinco pontos, que varia de $0-4$ ( $0=$ totalmente falso, $1=$ quase falso, $2=$ nem verdadeiro nem falso, $3=$ quase verdadeiro, $4=$ totalmente verdadeiro).

As respostas ao Inventário de Atitude Frente à Dor compõem escores para cada escala pela soma dos pontos referentes às respostas em cada item da escala em questão, divididos pelo número de itens respondidos. O escore final médio de cada escala poderá variar de 0 a 4 . Alguns itens têm sentido invertido e devem ter seus escores revertidos antes da soma, subtraindo-se de 4 o escore escolhido pelo entrevistado.

A interpretação do Inventário de Atitude Frente à Dor não é feita por ponto de corte, ou respostas certas ou erradas. Segundo o autor do inventário, o foco da análise deve ser a orientação das respostas mais "desejáveis" ou "menos desejáveis", por serem consideradas como mais ou menos adaptativas para o cuidado da dor crônica. A orientação desejável proposta pelo autor do inventário é a seguinte: controle $=4$; emoção $=4$; incapacidade $=0$; dano físico $=0$; medicação $=0$; solicitude $=0$ e cura médica $=0$ (Pimenta, 1999).

O conjunto de afirmativas que compõem cada um dos sete domínios busca aproximar-se das crenças do doente quanto: à influência pessoal no controle da dor, significando o quanto o doente acredita que pode controlar a sua dor (Controle); à relação entre emoção e intensidade da dor, o quanto o doente acredita que suas emoções influenciam na sua experiência dolorosa (Emoção); à dor como incapacitante ou o quanto o doente acredita que está incapacitado pela dor (Incapacidade); à dor como indicativo de dano físico, ou seja, o quanto o doente acredita que a dor significa que ele está "machucando" a si mesmo e que deveria evitar terapias com atividade física (Dano Físico); a medicamentos como melhor tratamento ou a crença que os medicamentos são o tratamento mais apropriado para dor crônica (Medicação); à solicitude de outros diante de pessoa com dor, o quanto o doente acredita que outros, especialmente os familiares devem ser mais solícitos quando ele sente dor (Solicitude); e, finalmen- 
te, ao quanto o doente acredita na cura médica para a sua dor ou na cura exclusivamente por meios médicos (Cura Médica) (Pimenta, 1999).

As entrevistas foram individuais, em sala privativa, por equipe devidamente treinada composta por uma médica do trabalho e dois fisioterapeutas, sem a presença de acompanhantes.

As informações quanto ao diagnóstico e história ocupacional foram obtidas no prontuário do CESAT e confirmadas com o trabalhador no momento da entrevista.

Para aplicação do IAD-breve foram explicados, a cada indivíduo, o objetivo do inventário e o modo de respondê-lo. Apesar de vários entrevistados demonstrarem boas condições de leitura e compreensão, optou-se pela leitura das assertivas e da escala de respostas, em voz alta, pelos entrevistadores, solicitando em seguida a resposta do entrevistado.

Ao final da entrevista foi feito o exame dos 18 pontos dolorosos (tender points) propostos como um dos critérios para a caracterização de fibromialgia.

Os dados foram organizados em banco de dados e analisados por meio do estatístico EPIINFO versão 6.0. Procedeu-se à análise a partir da comparação de médias das escalas do IAD-breve entre os indivíduos com diferentes atividades ocupacionais e as diversas variáveis socioculturais elencadas. Para variáveis dicotômicas utilizaram-se os testes t de Student e de Mann-Whitney e para as variáveis com mais de duas categorias aplicou-se a técnica de Análise de Variância (ANOVA) e o teste de KruskallWallis. A suposição de igualdade de variância requerida pela ANOVA foi verificada pelo teste de Bartlert. Para as variáveis que indicaram violação dos critérios para o uso da ANOVA foi utilizado o teste não-paramétrico de Kruskall Wallis.

As diferenças na composição dos grupos de atividades ocupacionais quanto às variáveis estudadas foram analisadas pelo Teste Chi-Quadrado para variáveis dicotômicas e ANOVA para variáveis contínuas, identificando-se que os grupos apresentam diferenças quanto à composição por sexo, escolaridade, tempo de exposição à sobrecarga ergonômica e renda. Adotou-se o nível de significância estatística de 5\% $(\mathrm{p}<0,05)$ para inferência estatística. As variáveis contínuas (tempo de exposição à sobrecarga ergonômica, tempo de dor, intensidade de dor e renda) foram analisadas utilizando-se os coeficientes de correlação de Pearson entre as escalas do IAD-breve e as variáveis.

\section{Resultados}

O grupo estudado constituiu-se de 65 trabalhadores com diagnóstico de LER/DORT de três atividades ocupacionais distintas: caixa bancário $(\mathrm{N}=22,33 \%)$, operador de caixa $(\mathrm{N}=18,27 \%)$, e industriário $(\mathrm{N}=25,38 \%)$. A composição do grupo foi semelhante à demanda do CESAT (Bahia, 2000). Predomina sexo feminino ( $\mathrm{N}=50,76,9 \%)$, com segundo grau completo $(\mathrm{N}=54,83,1 \%)$, empregados $(\mathrm{N}=32$, 49\%), com renda acima de dois salários mínimos $(\mathrm{N}=26,40 \%)$. A média de idade foi de 39 anos $(\mathrm{SD}=7,9)$ e a média de tempo de exposição à sobrecarga ergonômica foi de 11,4 anos $(\mathrm{SD}=6,2)$. Situações de litígio contra a empresa ou contra a previdência social ocorreram em $34,9 \%(\mathrm{~N}=22)$ e $65,1 \%$ têm prática religiosa (Quadro 1).

O sexo masculino foi predominante no grupo de industriários e não há homens entre os operadores de caixa. A escolaridade informada foi principalmente segundo grau completo, equivalendo a onze anos de estudo, principalmente para caixas bancários e operadores de caixa. Os caixas bancários apresentaram maior tempo de exposição à sobrecarga ergonômica e maior renda. Neste grupo apenas 29,5\% ( $\mathrm{N}=18)$ estão em litígio com a empresa ou com a previdência.

Quanto às características da dor, o grupo apresentou tempo médio de dor de cinco anos e intensidade média de 7,2 (Escala Numérica de 0 a 10). Cerca de 23,1\% apresentaram critérios para suspeita de fibromialgia (tender point acima de 11 pontos e alteração do sono).

A avaliação das médias do IAD-breve para o grupo e para cada atividade ocupacional (Quadro 2) mostra a orientação da atitude numa direção mais adaptativa para o domínio emoção $(2,35)$ apesar de estar próximo ao ponto neutro ou de mudança de atitude $(2,0)$. O domínio dano físico $(2,18)$ mostrou-se muito próximo ao ponto neutro $(2,0)$. Nas demais escalas observase uma orientação "não desejável" especialmente para os domínios controle $(1,83$, desejável=4), cura médica $(2,38$, desejável $=0)$, solicitude $(2,44$, desejável $=0)$ incapacidade $(3,20$, desejável=0) e medicação $(2,93$, desejável $=0)$.

A comparação das escalas segundo as atividades ocupacionais (Quadro 2) mostra diferença entre os três grupos no domínio dano físico, com uma orientação mais adaptativa para a atividade de operador de caixa $(1,59$, desejável $=0$ ), próxima ao ponto neutro para caixa 
Quadro 1

Descrição do grupo estudado quanto a variáveis demográficas, ocupacionais e culturais, Salvador, 2001.

\begin{tabular}{|c|c|c|c|c|c|c|}
\hline Co-variáveis & $\begin{array}{l}\text { aixa bancário } \\
\mathrm{N}=22\end{array}$ & $\begin{array}{l}\text { Operador de caixa } \\
\qquad N=18\end{array}$ & $\begin{array}{c}\text { Industriário } \\
\mathrm{N}=\mathbf{2 5}\end{array}$ & $\begin{array}{l}\text { População } \\
\text { total }\end{array}$ & $\begin{array}{c}\text { Chi-quadrado } \\
\text { p-valor }\end{array}$ & ANOVA \\
\hline Masculino & 5 & 0 & 10 & 15 & $0,0008^{*}$ & - \\
\hline Feminino & 17 & 18 & 15 & 50 & & \\
\hline Idade (média) & 40,9 & 37,1 & 38,0 & 39 & 0,26 & - \\
\hline \multicolumn{7}{|l|}{ Escolaridade } \\
\hline$<2^{\circ}$ grau & 0 & 1 & 10 & 11 & $0,0004^{*}$ & - \\
\hline $2^{\circ}$ grau completo e mais & is 22 & 17 & 15 & 54 & & \\
\hline \multicolumn{7}{|l|}{ Situação de trabalho } \\
\hline Empregado & 13 & 8 & 11 & 32 & 0,96 & - \\
\hline Desempregado & 9 & 10 & 14 & 33 & & \\
\hline \multicolumn{7}{|l|}{ Religião } \\
\hline Católica & 17 & 13 & 15 & 45 & 0,41 & - \\
\hline Outras & 5 & 5 & 10 & 12 & & \\
\hline \multicolumn{7}{|l|}{ Prática religiosa } \\
\hline Sim & 13 & 11 & 17 & 41 & 0,91 & - \\
\hline Não & 8 & 6 & 8 & 22 & & \\
\hline \multicolumn{7}{|l|}{ Local de nascimento } \\
\hline Capital & 11 & 11 & 11 & 33 & 0,53 & - \\
\hline Interior & 11 & 7 & 14 & 32 & & \\
\hline $\begin{array}{l}\text { Tempo de moradia } \\
\text { na capital (média) }\end{array}$ & 27 & 28 & 30 & 28,3 & - & 0,7 \\
\hline $\begin{array}{l}\text { Tempo de exposição } \\
\text { à sobrecarga } \\
\text { ergonômica (média) }\end{array}$ & 15,3 & 9,2 & 10,3 & 11,4 & - & $0,0002^{*}$ \\
\hline Tempo de dor & 5,5 & 4,3 & 5,0 & 5,0 & - & 0,38 \\
\hline $\begin{array}{l}\text { Renda } \\
\text { (média em salários } \\
\text { mínimos) }\end{array}$ & 5,2 & 1,88 & 2,3 & 3,1 & - & 0,38 \\
\hline \multicolumn{7}{|l|}{ Situação de litígio } \\
\hline Sim & 5 & 7 & 6 & 18 & 0,64 & - \\
\hline Não & 13 & 11 & 17 & 41 & & \\
\hline
\end{tabular}

${ }^{*} \mathrm{p}<0,05$

\section{Quadro 2}

Comparação das médias do Inventário de Atitude Frente à Dor, IAD-breve, para as atividades ocupacionais e população total do estudo, Salvador, 1999-2000.

\begin{tabular}{lccccc}
\hline Escalas & População & $\begin{array}{c}\text { Caixa bancário } \\
\text { total média }(\text { SD) }\end{array}$ & Operador de caixa & Industriário & $\begin{array}{c}\text { ANOVA } \\
(\mathbf{p}-\mathbf{v a l o r})\end{array}$ \\
\hline Controle & $1,83(0,84)$ & 1,87 & 1,92 & 1,74 & 0,7 \\
Emoção & $2,35(1,28)$ & 2,11 & 2,41 & 2,53 & 0,53 \\
Incapacidade & $3,20(0,9)$ & 3,09 & 3,27 & 3,26 & 0,76 \\
Dano físico & $2,18(1,54)$ & 2,08 & 1,59 & 2,67 & $0,01^{*}$ \\
Medicação & $2,93(1,04)$ & 3,0 & 2,87 & 2,92 & 0,92 \\
Solicitude & $2,44(1,13)$ & 2,17 & 2,73 & 2,38 & 0,29 \\
Cura médica & $2,38(0,57)$ & 2,37 & 2,4 & & 0,99
\end{tabular}

${ }^{*} \mathrm{p}<0,05$ 
bancário (2,08), e uma orientação menos adaptativa para industriários $(2,67)$. A comparação entre sexos mantém essa diferença no domínio dano físico com atitude mais adaptativa para sexo feminino $(1,98)$ e menos adaptativa para o sexo masculino $(2,83)$.

Embora o tipo de religião não tenha apresentado diferença na comparação das escalas do IAD-breve, ser praticante de alguma religião mostra uma orientação "mais adaptativa" para os domínios controle e emoção, sugerindo o quanto o doente acredita que pode controlar a sua dor e o quanto o doente acredita que suas emoções influem na sua experiência dolorosa (Quadro 4).

A comparação entre o grupo de trabalhadores afastados ou não do trabalho devido a LER/DORT não revela diferença entre as médias dos diversos domínios, assim como não houve correlação entre os escores do IAD-breve e as variáveis tempo de dor, tempo de exposição, intensidade de dor e renda.

A limitação para o trabalho, atividade doméstica, higiene pessoal, lazer e atividade sexual foram avaliadas com a atribuição de pontuação de 0 a 4 (nenhuma limitação, pouca limitação, limitação regular, limitação total). $\mathrm{O}$ quadro 5 mostra esta distribuição, com maior limitação do grupo para as atividades de lazer $(87,7 \%)$ e atividade doméstica $(76,9 \%)$ seguidas das atividades de higiene pessoal $(67,7 \%)$. Para atividade de trabalho, apenas 23,1\% $(\mathrm{N}=15)$ referiram maior limitação.

O quadro 6 mostra a comparação das escalas do inventário para limitação para o trabalho e atividades de higiene pessoal por apresentarem diferenças estatisticamente significantes entre os grupos com maior ou menor limitação. Na questão "limitação para o trabalho", o domínio dano físico mostra orientação "menos adaptativa" para o grupo que afirma ter menor limitação para o trabalho.

O grupo com maior limitação para as atividades de higiene pessoal apresenta uma orientação "mais desejável" para o domínio cura médica, o que indica uma crença menor na cura por meios médicos para sua dor, ou seja, a cura através de mecanismos externos ao doente. Para as questões limitação para atividade doméstica e atividade sexual, não houve diferença das médias do IAD-breve entre os que referiram maior ou menor limitação.

\section{Discussão}

A análise desses resultados remete inicialmente à discussão sobre a definição de quais as orientações "desejáveis" ou mais "adaptativas" para as atitudes diante da dor crônica. A proposta inicial dos autores deste inventário (Jensen et al., 1987; Jensen \& Karoly, 1992; Jensen et al., 1994) e suas modificações pela crescente utilização sugerem, segundo Pimenta (1999), que a decisão sobre qual a "melhor" orientação das atitudes diante da dor tenha de ser dinâmica, estabelecida para cada doente ou grupo de doentes, considerando: patologia, ambiente, tradições culturais e a "função" da atitude.

Esta autora problematiza que a dicotomia corpo-mente se expressa distintamente em diferentes contextos culturais. Esta dicotomia é mais acentuada, por exemplo, na cultura anglo-saxônica do que na latino-americana. Isto leva a diferentes interpretações quanto à percepção da influência da emoção na experiência dolorosa, sugerindo que essa orientação considerada desejável entre os primeiros pode não

Quadro 3

Comparação das médias do Inventário de Atitude Frente à Dor, IAD-breve, segundo sexo, Salvador, 1999-2000.

\begin{tabular}{lcccc}
\hline Escalas & Masculino & Feminino & ANOVA (p-valor) & K W (p-valor) \\
\hline Controle & 1,75 & 1,86 & 0,65 & 0,81 \\
Emoção & 2,18 & 2,41 & 0,55 & 0,57 \\
Incapacidade & 3,09 & 3,23 & 0,60 & 0,48 \\
Dano físico & 2,83 & 1,98 & $0,019^{*}$ & $0,02^{\star}$ \\
Medicação & 2,95 & 2,93 & 0,95 & 0,72 \\
Solicitude & 2,53 & 2,41 & 0,70 & 0,36 \\
Cura médica & 2,31 & 2,40 & 0,66 & 0,79 \\
\hline
\end{tabular}

${ }^{*} \mathrm{p}<0,05$ 
Quadro 4

Comparação das escalas do Inventário de Atitude Frente à Dor segundo prática religiosa.

\begin{tabular}{lcccc}
\hline Escalas & \multicolumn{2}{c}{ Praticante } & $\begin{array}{c}\text { ANOVA } \\
\text { (p-valor })\end{array}$ & $\begin{array}{c}\text { K W } \\
\text { (p-valor) }\end{array}$ \\
\hline Controle & Sim & Não & & \\
Emoção & 1,98 & 1,49 & 0,027 & $0,005^{*}$ \\
Incapacidade & 2,65 & 1,77 & 0,008 & $0,009^{*}$ \\
Dano físico & 3,12 & 3,31 & 0,45 & 0,26 \\
Medicação & 2,13 & 2,21 & 0,81 & 0,84 \\
Solicitude & 3,02 & 2,76 & 0,36 & 0,87 \\
Cura médica & 2,36 & 2,54 & 0,56 & 0,70 \\
\hline
\end{tabular}

${ }^{*} \mathrm{p}<0,05$

\section{Quadro 5}

Distribuição do grupo quanto à maior ou menor limitação.

\begin{tabular}{lccrc}
\hline Limitação & \multicolumn{2}{c}{ Maior } & N & Menor \\
\hline & N & $\%$ & 15 & 23,1 \\
\hline Atividade doméstica & 50 & 76,9 & 21 & 32,3 \\
Atividade higiene pessoal & 44 & 67,7 & 8 & 12,3 \\
Atividade de lazer & 57 & 87,7 & 50 & 76,9 \\
Atividade de trabalho & 15 & 23,1 & 28 & 43,1 \\
Atividade sexual & 37 & 56,9 & & \\
\hline
\end{tabular}

\section{Quadro 6}

Comparação das escalas do Inventário de Atitude Frente à Dor segundo limitação para o trabalho, atividades de higiene pessoal, domésticas e atividade sexual.

\begin{tabular}{lcccccc}
\hline Escalas & \multicolumn{3}{c}{ Limitação trabalho } & \multicolumn{3}{c}{ Limitação higiene pessoal } \\
\hline & Sim & Não & p-valor & Sim & Não & p-valor \\
\hline Controle & 2,18 & 1,73 & $0,07^{* *}$ & 1,76 & 1,97 & 0,37 \\
Emoção & 2,50 & 2,87 & 0,26 & 2,66 & 3,05 & 0,19 \\
Incapacidade & 2,32 & 2,58 & 0,15 & 2,53 & 2,5 & $0,44(\mathrm{KW})$ \\
Dano físico & 1,46 & 2,39 & $0,01^{*}$ & 2,26 & 1,99 & 0,40 \\
Medicação & 2,86 & 2,95 & 0,78 & 2,9 & 3,0 & 0,72 \\
Solicitude & 2,14 & 2,52 & 0,25 & 2,9 & 2,32 & 0,58 \\
Cura médica & 2,41 & 2,37 & 0,85 & 2,25 & 2,67 & $0,03^{*}$ \\
\hline
\end{tabular}

${ }^{*} \mathrm{p}<0,05$

${ }^{*} \mathrm{p}<0,1$ 
ser tão benéfica entre o latinos-americanos se a atitude de relacionar emoção à dor for vivenciada de modo que modificações do humor levem a respostas exacerbadas de comportamento doloroso (Pimenta, 1999).

Outro questionamento é sobre o domínio cura médica que pode ser benéfico por determinado tempo, podendo estimular o doente a envolver-se com determinadas propostas terapêuticas. De um modo geral, a autora sinaliza a imaturidade teórica dos domínios que compõem as atitudes diante da dor, seja na delimitação de seus conteúdos como na compreensão de como os conteúdos se articulam entre si e com outros conceitos circulantes (Pimenta, 1999). Apesar disso, reflete sobre a importância deste instrumento ao possibilitar a operacionalização de conceitos saídos da clínica dos doentes com dor. Assim, a proposta das orientações desejáveis será tomada como norteadora para a análise dos resultados deste estudo, embora com reflexões sobre outras possibilidades de interpretação.

A partir de observações clínicas, estudos qualitativos e quantitativos, a literatura sobre comportamento e dor enfatiza a associação entre um conjunto de crenças como: baixa capacidade de controlar a sua dor; a necessidade de respostas, por parte de familiares e outras pessoas, às solicitações; a associação da dor com dano tecidual; o acentuado sentimento de incapacidade pela dor e a crença na cura médica para a dor crônica; e uma maior disfunção psíquica, física e social, com interferência na adesão à terapêutica multidisciplinar que pressupõe a combinação de várias práticas terapêuticas não só medicamentosa, mas também abordagens corporais e psíquicas (Pimenta, 1999; Moore \& Brødsgaard, 1999).

Um outro aspecto importante é a associação entre o conhecimento sobre o diagnóstico e satisfação com o tratamento. Roth et al. (1998) discutem o quanto a incerteza e controvérsias sobre o diagnóstico em paciente com dor miofascial contribuem para crenças disfuncionais sobre a dor. Neste estudo, parte-se da hipótese que pacientes com dor miofascial quando comparados com um grupo de pacientes com dor crônica com diagnósticos menos controversos apresentam crenças não-adaptativas, tendendo a ampliar a gravidade dos sintomas, a prestar menor adesão e motivação ao tratamento e a considerar que a origem de sua dor é uma lesão estrutural mais grave. São, portanto, menos informados sobre seu diag- nóstico e a fonte patofisiológica da dor. Num grupo de 27 homens e 38 mulheres de uma clínica multidisciplinar de dor, Roth et al. (1998) identificam que apesar de pacientes com dor miofascial expressarem insatisfação com as terapias anteriores, apresentam mais insatisfação com a informação fornecida pelo médico no momento da consulta sobre seu diagnóstico e grau da lesão, tendendo a considerar que a sua dor representa algo "mais sério e diferente" do que o que foi informado pelo médico.

No atual estudo, a comparação das médias dos domínios para o grupo como um todo $(\mathrm{N}=65)$ revela que as médias dos domínios controle $(1,83$, desejável $=4)$, incapacidade $(3,2$, desejável=0) e medicação $(2,93$, desejável=0) apresentaram direção não desejável. Os domínios dano físico $(2,18)$, solicitude $(2,44)$ e cura médica $(2,38)$ estiveram mais próximos do ponto neutro (ponto neutro=2) e o domínio emoção $(2,35)$ também esteve numa direção mais adaptativa (Quadro 2).

A comparação entre os resultados deste estudo com os de um estudo brasileiro que avaliou 69 pacientes em São Paulo com dor crônica de origem neuropática, oncológica e muscular (Pimenta, 1999), e outro de origem norteamericana, que avaliou 94 doentes com dor crônica no início do tratamento, investigando com diferenças étnicas (Jensen \& Karoly ,1994 in Pimenta, 1999), mostra algumas semelhanças e diferenças.

Para os domínios controle e dano físico os três estudos mostraram resultados semelhantes sendo o domínio controle numa direção não desejável. Portanto, para os três grupos de doentes há um predomínio das crenças de que o doente não pode controlar sua dor. O domínio dano físico foi orientado para o ponto neutro ou de mudança de atitude. Para os domínios medicação e cura médica os dois estudos brasileiros mostraram uma direção menos adaptativa reforçando a idéia que medicação é o melhor tratamento para dor crônica e que esta poderá ser curada pela ação médica. Para os domínios "solicitude" e incapacidade o estudo de Jensen \& Karoly (1994) e Pimenta (1999) apresentaram resultados semelhantes e numa direção mais desejável que o atual estudo.

Em contraste com a avaliação da média geral do grupo, a comparação das escalas do inventário entre as atividades ocupacionais e sexo revela diferenças entre as médias do domínio dano físico numa direção mais desfavorável para os industriários, sexo masculino e me- 
nor limitação para o trabalho. Este resultado pode ser entendido na perspectiva do autor deste inventário como se este grupo de trabalhadores da operação e manutenção da indústria acreditassem que a dor representa dano físico e que por isso poderiam ter menor disposição para aderir a tratamentos que implicassem atividade física. Por outro lado, este grupo também corresponde a sujeitos que estão inseridos em processos de trabalho que exigem maior "esforço físico" e que, portanto, é possível pensar que a experiência do sintoma doloroso poderá ter sido marcada pela associação com as atividades exercidas na indústria e não com atividades físicas terapêuticas.

A diferença de médias do IAD-breve entre sexos, com uma direção mais desfavorável para os homens no domínio dano físico (Quadro 3), reforça o raciocínio anterior, porque a maioria dos indivíduos do sexo masculino $(\mathrm{N}=15)$ está no grupo de industriários $(\mathrm{N}=10)$ e em menor número entre os bancários $(\mathrm{N}=5)$. Em artigo de Jensen et al. (1987), que apresenta uma primeira versão do IAD validada, os autores mostram diferença nas respostas entre homens e mulheres sugerindo sub-escalas para os domínios "medicação, incapacidade e controle" e não comentam sobre o domínio "dano físico".

A média do domínio dano físico numa direção mais desejável $(1,46$, desejável $=0)$ entre os que referiram maior limitação para o trabalho sugere inicialmente uma contradição, porque reforça a idéia, segundo Jensen, de que estes sujeitos evitariam exercícios físicos, mas também pode significar que o grupo que identifica maior limitação para o trabalho encontrase mais engajado no processo terapêutico, concordando mais com as assertivas que "os exercícios físicos podem diminuir a intensidade da dor" ou "são bons para o problema da dor", e portanto poderá estar experimentando os benefícios da atividade física terapêutica para a melhoria dos sintomas físicos e emocionais, distinguindo este tipo de atividade física da sobrecarga ergonômica no processo de trabalho.

Por outro lado, é importante salientar que a referência à maior limitação para o trabalho ocorreu em apenas 15 trabalhadores entrevistados (23\%), diferentemente das respostas quanto à limitação para atividade de lazer $(87,7)$, atividade doméstica $(76,9 \%)$, atividade de higiene pessoal $(67,7 \%)$ e atividade sexual $(56,9 \%)$. Este dado pode estar influenciado pelo local da entrevista, no CESAT, que pode ser visto pelos trabalhadores como uma instituição que tem alguma influência sobre sua situação previdenciária.

Apesar de a diferença para o domínio "emoção", entre praticantes de religião, não ter sido encontrada em outros estudos, o sentido da média neste domínio sugere uma maior compreensão da relação entre dor e emoção e é apontada na literatura como uma orientação que pode auxiliar o doente com dor a adquirir recursos para melhor lidar com a dor (Pimenta, 1995; 1999; Turner, 1996; Pimenta et al., 1997). O resultado desse estudo sinaliza a possibilidade que entre os praticantes de religião haja uma maior atenção em relação às variações da emoção e a piora ou melhora da dor reforçando a idéia que a prática religiosa poderá influenciar no processo de autoconhecimento ou de aceitação da dor.

A questão da aceitação da dor como doença crônica parece contribuir para o melhor funcionamento do indivíduo. Segundo Pimenta (1999), os doentes que julgam sua dor inaceitável fazem tudo para evitá-la a qualquer custo; expõem-se com maior facilidade a tratamentos agressivos, procuram atendimento em diversas clínicas e, muitas vezes, perdem a oportunidade de continuar vivendo as suas vidas apesar da dor (1999). Outros estudos apontam nessa direção (McGracken, 1998; Bates \& Rankin-Hill, 1994), ao apresentar o processo de aceitação da dor como constituído por fases (pré-contemplação, contemplação, preparação, ação e manutenção), sugerindo que a assistência a esses doentes incorpore o conhecimento sobre essas fases; e ao observar o processo de cada sujeito doente, apoiando-o no processo de mudança de perspectiva no sentido da reorganização do seu mundo da vida (Schutz, A. in Capaldo, 1979).

A relação médico-paciente e a qualidade da comunicação nesta díade (Turner, 1996; Roth et al., 1998) têm mostrado significativa influência sobre os sintomas do paciente com dor. Nos casos de dor crônica, tem sido amplamente reconhecida a maior influência das expectativas do paciente e a interação deste com o terapeuta sobre os resultados terapêuticos do que os tipos de tratamento especificamente aplicados. Apesar de chamar a atenção para a necessidade de investigar mais a fundo a eficácia terapêutica de diversos tratamentos utilizados nos casos de dor, especialmente aqueles que impliquem uma maior exposição a riscos. 


\section{Conclusão}

Conhecer a constelação de atitudes frente à dor de trabalhadores com diagnóstico de LER/ DORT foi a motivação inicial deste estudo, que possibilitou conhecermos também um pouco mais sobre as variações de atitudes que pareciam compor um grupo semelhante. A orientação da psicologia cognitivo-comportamental oferece alguns instrumentos que podem ser utilizados de forma mais sistemática nesta população de trabalhadores e orientar abordagens individuais e coletivas, possibilitando aos profissionais de saúde conhecerem diferenças de atitudes e comportamentos em subgrupos, contribuindo para o melhor planejamento dos recursos terapêuticos.

Os resultados do domínio "dano físico" para sexo masculino, industriários e trabalhadores que referem maior limitação para o trabalho merecem maior aprofundamento com a ampliação da população de estudo e associação de outros recursos metodológicos. A prática religiosa e os domínios "emoção" e "controle" também apresentam plausibilidade com a teoria cognitivo-comportamental e justifica o investimento em outros estudos no sentido de melhor conhecer estas variações de atitudes e dor em trabalhadores.

O reconhecimento da dor crônica como um foco importante de cuidado em trabalhadores com LER/DORT amplia a necessidade de diálogo com a clínica a partir de uma maior compreensão da complexidade do fenômeno da dor crônica, assim como a necessidade de expandir o conceito da assistência e reabilitação desses trabalhadores tendo como referência um modelo de cuidado integral. O papel dos centros de referência à saúde dos trabalhadores na relação com a rede de assistência e reabilitação deverá sofrer modificações na direção de uma ação mais proativa de capacitação, atualização e organização desta demanda.

\section{Colaboradores}

MAG Lima é responsável pela concepção da idéia inicial, definição do escopo do trabalho e das abordagens metodológicas, pesquisa bibliográfica, construção e aplicação dos instrumentos (entrevistas), análise, redação e revisão do artigo. R Neves trabalhou na discussão do escopo do projeto, aperfeiçoamento e aplicação dos instrumentos (entrevistas), pesquisa bibliográfica, digitação, limpeza do banco, análise e revisão da redação; S Sá, na pesquisa bibliográfica, discussão e aperfeiçoamento dos instrumentos, entrevista e digitação. C Pimenta foi a orientadora desde a fase inicial no projeto e colaborou na discussão e aperfeiçoamento da abordagem teórico-metodológica, pesquisa bibliográfica, discussão e aperfeiçoamento dos instrumentos, discussão dos resultados, análise dos dados e revisão do artigo.

\section{Agradecimentos}

Agradecemos a todos os trabalhadores atendidos pelo CESAT e em especial àqueles que gentilmente aceitaram participar deste estudo, à direção do CESAT, na pessoa da doutora Letícia Nobre pelo incentivo; à doutora Cibele Pimenta pela paciente orientação e atenção; ao doutor Durval Kraychete pela oportunidade de aprofundar nossos conhecimentos sobre dor crônica e a todos que direta ou indiretamente contribuíram para a realização deste estudo. 


\section{Referências bibliográficas}

Bahia 2000. Secretaria da Saúde do Estado da Bahia. Centro de Estudos da Saúde do Trabalhador. Estudo da demanda: DORT/LER, 1999. COAST/CESAT, Salvador.

Bates MS, Edwards WT \& Anderson KO 1993. Ethnocultural influences on variation in chronic pain perception. Pain 52:101-112.

Bates MS \& Lesley R-H 1994. Control, culture and chronic pain. Society of Science and Medicine 39(5):629-645.

Bates MS, Rankin HL, Sanchez AM \& Mendez BR 1995. A cross-cutural comparison of adaptation to chronic pain among Anglo-Americans and native Puerto Ricans. Medical Anthropology 16:141-173.

Capalbo C 1979. Metodologia das ciências sociais. A fenomenologia de Alfred Schutz. Antares Universitária. Rio de Janeiro.

Helman CG 1994. Cultura, saúde e doença. (3a ed.). Artes Médicas, Porto Alegre.

Kleinman A 1987. Anthropology and psychiatry. The role of culture in cross-cultural research on illness. $\mathrm{Br}$. Journal Psychiatry 151:447-454.

Kleinman A, Eisenberg L \& Good B 1978. Culture, illness, and care: clinical lessons from anthropologic and cross-cultural research. Ann. Intern. Med. 88:251-258.

Jensen MP \& Karoly P 1992. Pain-specific beliefs, perceived symtom severity, and adjustment to chronic pain. The Clinical Journal of Pain 123-130.

Jensen MP, Karoly P \& Huger R 1987. The development and preliminary validation of an instrument to assess patients attitudes toward pain. Journal of Psychosomatic Research 31(3):393-400.

Jensen MP \& Karoly P 1991. Control beliefs, Coping efforts, and adjustment to chronic pain. Journal of Consulting and Clinical Psychology 59(3):431-438.

Jensen MP, Turner JA, Romano JM \& Lawler BK 1994. Relationship of pain-specific beliefs to chronic pain adjustment. Pain. The Journal of the International Association for the Study of Pain 57:301-309.
McGracken LM 1998. Learning to live with pain: acceptance of pain predicts adjustment in persons with chronic pain. Pain 74(1):21-7.

Melzack R \& Wall PD 1965. Pain mechanisms: a new theory. Science 5:971-979.

Moore R \& Brødsgaard I 1999. Cross-cultural investigator of pain, pp. 53-80. In Epidemiology of Pain. I.K. IASP Press, Crombie.

Pimenta CAM 1995. Aspectos culturais e a experiência dolorosa. Revista da Escola de Enfermagem da Universidade de São Paulo 32(2):179-186.

Pimenta CAM, Koizumi MS \& Teixeira MJ 1997. Dor, depressão e conceito culturais. Arq. Neuropsiquiatria 55(3-A):370-380.

Pimenta CA 1999. Atitude de doentes com dor crônica frente à dor. Tese de livre-docência. Escola de Enfermagem. Universidade de São Paulo.

Roth RS, Horowitz K \& Bachman JE1998. Chronic myofascial pain: knowledge of diagnosis and satisfaction with treatment. Archives of Physical Medicine and Rehabilitation 79(8):966-970.

Tait RC \& Chibnall JT 1997. Development of a brief version of the Survey of Pain Attitudes. Pain 70(2/3): 229-235.

Turner AJ 1996. Does a successful outcome imply an effective treatment?, pp. 153-159. In Pain treatment center at a crossroads: a practical and conceptual reappraisal, progress in pain research and management. M.J.M Cohen and J.N.Campbell, IASP Press, Seattle.

Zoborowski M 1969. People in pain. Jossey-Bass, San Francisco.

Zola IK 1966. Culture and symtoms - An analisys of patients presenting complaints. American Sociological Review 31:615-630.

Artigo apresentado em 10/12/2003

Aprovado em 20/05/2004

Versão final apresentada em 30/5/2004 